\title{
Management von Patienten mit Vorhofflimmern und intrakraniellen Blutungen: Ist eine erneute Antikoagulation möglich? Die ENRICH-AF Studie
}

\author{
H.-C. Diener ${ }^{1}$, M. Endres ${ }^{2}$ \\ ${ }^{1}$ Institut für Medizinische Informatik, Biometrie und Epidemiologie (IMIBE), Medizinische Fakultät der Universität Duisburg-Essen, Essen, \\ Deutschland \\ ${ }^{2}$ Klinik und Hochschulambulanz für Neurologie, Charité - Universitätsmedizin Berlin, Berlin, Deutschland
}

\section{Einleitung}

Patienten mit Vorhofflimmern haben ein hohes Risiko, einen ischämischen Schlaganfall zu erleiden. Dieses Risiko kann durch eine orale Antikoagulation mit Vitamin-K-Antagonisten oder direkten oralen Antikoagulantien (DOAK) um 60 bis 80\% reduziert werden. Eine typische und sehr gefürchtete Komplikation der oralen Antikoagulation ist eine intrakranielle Blutung. Wenn diese Blutung überlebt wird, stellt sich die Frage, ob eine erneute Antikoagulation möglich und notwendig ist. Aber auch bei Patienten mit Zustand nach Hirnblutung und zusätzlichem Vorhofflimmern, die bislang nicht antikoaguliert wurden, stellt sich die Frage, ob diese neu begonnen werden soll. Die Datenlage zu diesem Problem soll im Folgenden kurz dargestellt werden.

\section{Risiko einer erneuten intrazerebralen Blutung nach Wiederaufnahme der oralen Antikoagulation}

Das Risiko einer erneuten intrazerebralen Blutung bei Patienten mit Vorhofflimmern, die wieder antikoaguliert werden, liegt zwischen 2,5 und 8\%/Jahr und im Mittel bei 2,6\% [7]. In Beobachtungsstudien hatten Patienten, die nach einer intrazerebralen Blutung erneut antikoaguliert wurden, kein höheres Risiko einer erneuten intrazerebralen Blutungen als Kontrollen [10]. Eine Metaanalyse von acht Studien mit 5.306 Patienten mit Vorhofflimmern nach intrazerebraler Blutung zeigte, dass die Wiederaufnahme der Antikoagulation mit einem signifikant geringeren Risiko für thromboembolische Komplikationen verbunden war (Risiko: 0,34; 95\%-Konfidenzintervall: $0,25-0,45 ; \quad Q=5,12, \quad P$ für Heterogenität $=0,28$ ). Es gab keinen Hinweis auf ein erhöhtes Risiko für eine erneute intrazerebrale Blutung nach Wiederaufnahme der Antikoagulationstherapie, obwohl es eine signifikante Heterogenität zwischen den eingeschlossenen Studien gab (relatives Risiko: 1,01; 95\%-Konfidenzintervall: $0,58-1,77 ; \mathrm{Q}=24,68, \mathrm{P}$ für Heterogenität $<0,001$ [ [8]. Dabei muss aber berücksichtigt werden, dass die betreuenden Ärzte Patienten, bei denen sie ein hohes Risiko einer Rezidivblutung unterstellen, nicht erneut antikoagulieren. Daher sind dringend prospektive randomisierte Therapiestudien notwendig.

\section{Risikofaktoren für eine erneute intrazerebrale Blutung}

Es gibt eine Reihe von Risikofaktoren für eine erneute intrazerebrale Blutung. Diese fließen in die Entscheidung ein, ob eine erneute orale Antikoagulation möglich ist. Die wichtigsten Prädiktoren sind große intrazerebrale Blutung, lobäre Blutung, ausgeprägte zerebrale Mikroangiopathie, zahlreiche Mikroblutungen in der Kernspintomografie, eine Amyloidangiopathie, fortgeschrittene Lebererkrankungen und Übergebrauch von Alkohol [6]. Ein erhöhtes Risiko von Stürzen erhöht auch das Risiko für traumatische subdurale Hämatome. Leider ist immer noch ungeklärt, ab welcher Zahl von zerebralen Mikroblutungen das Risiko einer erneuten intrazerebralen Blutung unter Antikoagulation erhöht ist.

\section{Zeitpunkt der erneuten Antikoagulation}

Bisher ist durch randomisierte Studien nicht geklärt, welches der ideale Zeitraum ist, zu dem eine orale Antikoagulation wieder aufgenommen werden kann. Erfolgt diese zu früh, besteht das Risiko einer erneuten intrazerebralen Blutung, erfolgt sie zu spät, ist das Risiko einer thromboembolischen Komplikation insbesondere eines ischämischen Schlaganfalls erhöht. Die meisten Registerstudien zeigen, dass eine erneute orale Antikoagulation in einem Zeitraum zwischen 2 und 8 Wochen nach der initialen Blutung möglich ist [9]. In der deutschen Kohorten Studie mit 719 Patienten nach intrazerebraler Blutung und Vorhofflimmern wurde die orale Antikoagulation im Mittel nach 1 Monat wieder aufgenommen [5].

\section{Welche Antikoagulantien sollten eingesetzt werden?}

Die meisten bisher durchgeführten Registerstudien erfolgten bei Patienten, die erneut mit Vitamin-K-Antagonisten antikoaguliert worden. Die DOAKs haben im 
Verhältnis zu Vitamin-K-Antagonisten ein deutlich reduziertes Risiko einer intrazerebralen Blutung [2]. Außerdem gibt es sowohl für Dabigatran wie für die Faktor XaAntagonisten in der Zwischenzeit spezifische Antidota [1], die die Mortalität bei intrazerebralen Blutungen um $30-50 \%$ reduzieren können [4].

\section{Laufende Therapiestudien}

Der interventionelle Verschluss des linken Vorhofohres ist eine Therapieoption bei Patienten mit intrazerebralen Blutungen, bei denen Kontraindikationen für eine langfristige orale Antikoagulation bestehen. Die STROKE-CLOSE Studie in Skandinavien vergleicht hierzu den Verschluss des linken Vorhofohrs gegen die beste verfügbare konservative Therapie. In die deutsche CLOSURE-AF Studie, die vom Deutschen Zentrum für Herz-Kreislaufforschung (DZHK) finanziert wird, werden unter anderem auch Patienten mit Vorhofflimmern nach intrazerebraler Blutung aufgenommen [3]. Insgesamt fünf prospektive Studien untersuchen derzeit den Einsatz von DOAKs im Vergleich $\mathrm{zu}$ anderen Therapien bei Patienten mit intrazerebraler Blutung und Vorhofflimmern. In Deutschland läuft im Moment auch die EU-finanzierte PRESTIGE-AF Studie. Hier wird ein NOAK mit Thrombozytenfunktionshemmern oder keiner antithrombotischen Therapie verglichen.

\section{Die ENRICH-AF Studie - Studienzentren gesucht}

Die ENRICH-AF Studie untersucht, ob Edoxaban in einer Dosis von 60 oder $30 \mathrm{mg}$ täglich verglichen mit Thrombozytenfunktionshemmern oder keiner antithrombotischen Therapie das Risiko von erneuten Schlaganfällen bei Patienten nach intrakranieller Blutung verhindert. Die Studie findet auch in Deutschland statt und wird geleitet von Prof. Dr. Matthias Endres von der Charité in Berlin und Prof. Dr. Hans-Christoph Diener von der Abteilung für Neuroepidemiologie an der Universität Duisburg-Essen. Die Studie schließt Patienten mit Vorhofflimmern im Alter über 45 Jahre ein, die eine intrakranielle Blutung (d.h. intrazerebral, subdural oder subarachnoidal, aber keine eingebluteten Hirninfarkte) erlitten haben. Ein Studieneinschluss ist frühestens 14 Tage nach dem Ereignis möglich. Der primäre Endpunkt der Studie ist die Kombination aus ischämischem und hämorrhagischem Schlaganfall und einem Schlaganfall ungeklärter Ätiologie. Der wichtigste Sicherheitsendpunkt ist die Häufigkeit von schwerwiegenden Blutungskomplikationen nach den ISTH Kriterien. In einer kernspintomografischen Substudie soll geklärt werden, welche Rollen eine fortgeschrittene Mikroangiopathie und zerebrale Mikroblutungen für die Prognose spielen beide stellen keine Kontraindikation für den Studieneinschluss dar.

Wir suchen derzeit insbesondere Rehabilitationskliniken, in denen Patienten mit Vorhofflimmern nach intrakranieller Blutung behandelt werden und die Interesse an einer Teilnahme an dieser Studie haben.

Interessenten wenden sich bitte an Prof. Endres unter der E-Mail-Adresse: matthias.endres@charite.de

\section{Literatur}

1. Alonso A, Diener H. Medikamentöse Therapie bei akuten Hirnblutungen unter Antikoagulation. Arzneimitteltherapie 2021; 39(5): 144-58.

2. Chatterjee S, Sardar P, Biondi-Zoccai G, Kumbhani DJ. New oral anticoagulants and the risk of intracranial hemorrhage: Traditional and Bayesian meta-analysis and mixed treatment comparison of randomized trials of new oral anticoagulants in atrial fibrillation. JAMA Neurol 2013; 70(12): 1486-90.

3. Häusler KG, Endres M, Landmesser U. [Left atrial appendage occlusion in patients with nonvalvular atrial fibrillation : Present evidence, ongoing studies, open questions]. Med Klin Intensivmed Notfmed. 2020; 115(2): 107-13.

4. Kermer P, Eschenfelder CC, Diener HC, Grond M, Abdalla Y, Abraham A, et al. Antagonizing dabigatran by idarucizumab in cases of ischemic stroke or intracranial hemorrhage in Germany-Updated series of 120 cases. Int I Stroke 2020: 1747493019895654.

5. Kuramatsu JB, Gerner ST, Schellinger PD, Glahn J, Endres M, Sobesky J, et al. Anticoagulant reversal, blood pressure levels, and anticoagulant resumption in patients with anticoagulation-related intracerebral hemorrhage. JAMA 2015; 313(8): 824-36.

6. Li YG, Lip GYH. Anticoagulation Resumption After Intracerebral Hemorrhage. Curr Atheroscler Rep 2018; 20(7): 32.

7. Lip GY, Frison L, Halperin JL, Lane DA. Comparative validation of a novel risk score for predicting bleeding risk in anticoagulated patients with atrial fibrillation: the HAS-BLED (Hypertension, Abnormal Renal/ Liver Function, Stroke, Bleeding History or Predisposition, Labile INR, Elderly, Drugs/Alcohol Concomitantly) score. J Am Coll Cardiol 2011; 57(2): 173-80.

8. Murthy SB, Gupta A, Merkler AE, Navi BB, Mandava P, ladecola C, et al. Restarting Anticoagulant Therapy After Intracranial Hemorrhage: A Systematic Review and Meta-Analysis. Stroke 2017; 48(6): 1594-600.

9. Nielsen PB, Larsen TB, Skjoth F, Gorst-Rasmussen A, Rasmussen LH, Lip GY. Restarting Anticoagulant Treatment After Intracranial Hemorrhage in Patients With Atrial Fibrillation and the Impact on Recurrent Stroke, Mortality, and Bleeding: A Nationwide Cohort Study. Circulation 2015; 132(6): 517-25.

10. Nielsen PB, Larsen TB, Skjoth F, Lip GY. Outcomes Associated With Resuming Warfarin Treatment After Hemorrhagic Stroke or Traumatic Intracranial Hemorrhage in Patients With Atrial Fibrillation. JAMA Intern Med 2017; 177(4): 563-70.

\section{Acknowledgements}

M.E. received funding from DFG under Germany's Excellence Strategy EXC-2049 - 390688087, BMBF, DZNE, DZHK, EU, Corona Foundation, and Fondation Leducq

\section{Interessenvermerk}

HCD und ME sind die deutschen Studienleiter der ENRICH-AF Studie. Für diese Tätigkeit erhalten sie keine Vergütung.

ME reports grants from Bayer and fees paid to the Charité (no personal fees) from Amgen, AstraZeneca, Bayer, Boehringer Ingelheim, BMS, Covidien, Daiichi Sankyo, GSK, Novartis, Pfizer, Sanofi, all outside the submitted work.

\section{Korrespondenzadresse:}

Prof. Dr. Hans Christoph Diener

Institut für Medizinische Informatik, Biometrie

und Epidemiologie (IMIBE)

Medizinische Fakultät der Universität Duisburg-Essen

Hufelandstraße 55

D-45147 Essen

hans.diener@uk-essen.de 